\title{
Verkehrsplanung am Scheideweg?
}

\author{
Die Bilanzierung der kommunalen Verkehrsplanung zeigt, daß sie das Problem \\ des Verkehrs in den deutschen Städten nicht hat lösen können. Die tatsächli- \\ chen Praxiserfolge im Verkehrssektor fallen wesentlich geringer aus, als es \\ ihre theoretische und öffentliche Rezeption vermuten läßt. Gleichzeitig droht \\ die Tatsache, daß der Verkehr ein gewichtiges Problem für Städte und Regio- \\ nen darstellt, unfer dem Druck der ökonomischen Rahmenbedingungen von der \\ Tagesordnung in Politik und Planung abgesetzt zu werden.
}

\section{$\mathrm{D}$} Von Heike Flämig e aktuelle Entwicklung im Bereich der Verkehrspolitik ist durch einen sich zuspitzenden Konflikt gekennzeichnet. Es besteht eine große Diskrepanz zwischen den wachsenden Ansprüchen an Umweltverträglichkeit, soziale Ausgewogenheit und Gestaltbarkeit des Verkehrs und den Umsetzungsgrenzen einer dafiir notwendigen Politik.

Eine problemorientierte Analyse von Mobilität und Verkehr kommt insbesondere in den Verdichtungsräumen $\mathrm{zu}$ folgendem thesenartigen Befund(1):

- Die Verkehrsmengenentwicklung ist weiterhin steigend und problematisch, wenn diese auch nicht zum vielfach prognostiziertem Zusammenbruch (Verkehrsinfarkt) geführt hat.

- Kritisch stellen sich heute weniger die Luftemissionen dar (technisch scheint das Problem mittelfristig lösbar), als vielmehr die strukturellen Folgen (Lärm, Flächenverbrauch, Lebensraumzerschneidung).

- Mit Blick auf die soziale und ökonomische Dimension der Nachhaltigkeit bildet die Mobilität einen weiterhin ungelösten Konflikt für die Städte (Beispiele sind das Problem der Autoabhängigkeit und das Problem der Bezahlbarkeit der Verkehrssysteme für die Gesellschaft aber auch der Verkehrsmittel durch die Individuen). Im Kontext des technologischen, sozialen und ökonomischen Wandels verändern sich Problemwahrnehmung und Prioritäten der Stadtverkehrspolitik. Wichtigste Faktoren sind vor allem die „leeren Kassen“ der öffentlichen Hand, die beispielsweise eine flächenhafte Verkehrsberuhigung mit flankierenden Rückbaumaßnahmen nicht mehr möglich machen, und die allgemein angespannte Wirtschaftslage, die eine Prioritätsverschiebung der Argumentationslinien von „ökologiefundiert" zu „ökonomiebe- stimmt" bewirkt. Verstärkt wird dieser Trend durch das (bisherige) Ausbleiben des ,Verkehrsinfarkts".

Das höchst unterschiedliche Anpassungsverhalten der Mobilen an veränderte Bedingungen trägt scheinbar zu einer effizienten Selbstregulation des Verkehrssystems bei. Unbeabsichtigte und geplante Ausnahmesituationen (Unfälle, Staus, Baustellen) demonstrieren immer wieder, wie groß das Anpassungsvermögen der Verkehrsbeteiligten tatsächlich ist. Dies muß nun nicht zwangsläufig gerecht oder ökologisch sein, aber es erhält die Funktionsfähigkeit des Verkehrssystems aufrecht.

\section{- Das "neve Politikmodell“}

Die bisherige kommunale Verkehrsplanungspraxis läßt sich durch zwei Paradigmen kennzeichnen (2). Bis Ende der 70er Anfang der 80er war es üblich General- bzw. Gesamtverkehrspläne (GVP) zu erstellen. Im GVP bilden die Verkehrsmittel das Subjekt des Entwicklungsprozesses, die Infrastruktur und die Stadtentwicklung die Objekte. Im GVP ist die Planung am Nachvollzug der Verkehrsentwicklung ausgerichtet, vor allem derjenigen des Autoverkehrs. Die heute ,gängigen“ Verkehrsentwicklungspläne (VEP) fokussieren auf die Stadt, zu deren Entwicklung auch eine entsprechende Planung des Verkehrs beitragen soll. Im VEP ist die gewünschte Stadtentwicklung die Leitgröße und das Verkehrssystem Objekt der Planung. Die kommunale Verkehrsentwicklungsplanung umfaßt das gesamte Planungsgebiet, behandelt alle Verkehrsarten und zielt auf eine Integration der Ansprüche in Produkt und Prozeß. Es wird von einer integrativen oder auch integrierten Verkehrsplanung gesprochen, die mehr sein soll, als die „Addition sektoraler Fachplanungen und die bloße Verknüpfung von Verkehrsteilsy- stemen (Straße, Schiene, Luft, Wasserweg), sondern der offene Prozeß einer aufeinander abgestimmten Planung von Siedlungsstrukturen und Verkehr" (3).

Bei der Betrachtung von umgesetzten Beispielen kommunaler und regionaler Verkehrsplanung (4) zeigt sich ein beachtlich breites Spektrum an Maßnahmen zur Verkehrsgestaltung, vorrangig in verkehrsträgerbezogenen Handlungsfeldern wie Infrastruktur, Netzplanung, Verkehrsangebote. Eine umfassende Berücksichtigung aller Verkehre nach Beförderungszwecken und -objekten fehlt jedoch. Die meisten verkehrsplanerischen Maßnahmen zielen auf die Beeinflussung des Personenverkehrs, mit einem Schwerpunkt bei den Berufsverkehren. Maßnahmen im Bereich des Freizeitverkehrs sind eher unterentwickelt. Wenn Maßnahmen im Bereich des Güterverkehrs umgesetzt werden, handelt es sich in der Regel um harte Infrastrukturmaßnahmen. Oder es wird nach abstrakten bzw. komplexen Lösungen (z.B. Güterverkehrszentren) gesucht, deren ökologische und ökonomische Wirkungen noch nicht abschließend beurteilt werden können.

Zunehmend kommen auf der kommunalen und betrieblichen Handlungsebene allerdings auch Marketing und andere „weiche" Instrumente zur Anwendung, wie Formen von kooperativen Planungsprozessen oder etwa zur Gestaltung der Öffentlichkeitsarbeit. Damit ist in jüngster Zeit ein Tendenzwandel vom Planen und Bauen in Richtung Regeln und Vermitteln feststellbar. „Mobilitätsmanagement" entwickelt sich in diesem Kontext zunehmend zur Basisstrategie der kommunalen Verkehrsentwicklung(-splanung). Auf der Ebene der Unternehmen entsteht gleichzeitig ein neues Handlungsfeld des ,betrieblichen Mobilitätsmanagements", das die am Unternehmensstandort erzeugte Verkehrsnachfrage (Berufsverkehr, Wirtschaftsverkehr) zum Gegenstand von Optimierungsstrategien macht. Die bisher realisierten Beispiele haben teilweise durchaus Vorbildcharakter, sie sind andererseits aber bezogen auf ihre Dauer und Verbindlichkeit noch sehr labil.

In räumlicher Hinsicht gilt die bisherige Praxis nur für die kommunale Ebene. Politikstrategien, die auf regionaler Ebene vorgefunden werden können, beziehen sich in der überwiegenden Zahl der Fälle auf Fragen der Flächennutzung (Gewerbeplanung, großflächige Standorte), der Wirt- 
schaftsförderung und der institutionellen Kooperation. Gemeinsame Verkehrsplanungsaktivitäten sind die Ausnahme bzw. werden erst im Zuge der Regionalisierung des Nahverkehrs initiiert.

\section{- Begrenzte Wirksamkeif}

Aus der Recherche ist neben vordergründigen Erfolgsfällen deutlich geworden, daß die bisher praktizierte Verkehrsplanung das Problem des Verkehrs in Städten nicht hat lösen können. Bereits das alte Politikmodell $(=$ mehr Wege für mehr Verkehr) ist an diesem Anspruch gescheitert. Ob die modernisierte Stadtverkehrsplanung der 80er Jahre faktisch - und nicht nur normativ - überzeugender war, ist im Licht der analysierten Beispielsfälle noch offen. Es wurde viel gebaut. Verhaltensmodifikationen als Ausgangspunkt einer spürbar veränderten Verkehrsorganisation sind offensichtlich in viel geringerem Ausmaß erzielt worden, als eigentlich beabsichtigt war oder aus Sicht der Nachhaltigkeit notwendig wäre. Die Wirkungstiefe der realisierten Maßnahmen nimmt, betrachtet man Effekte in den Sektoren Städtebau, Verkehr und Umwelt, in genau dieser Reihenfolge systematisch $a b$. Derzeit weist alles darauf hin, daß zwar Städte umgebaut, nicht aber der Verkehr verändert wurde.

Es bleibt die Frage, worauf diese geringen Praxiserfolge im Verkehrssektor zurückzufuihren sind. Gibt es in programmatisch-konzeptioneller Hinsicht mittlerweile eine solide Fundierung von Politikstrategien im Bereich Mobilität, treten zunehmend Diskrepanzen zwischen Wissen und Handeln, Planung und Realität auf (,Umsetzung"). Viele vermeintliche Lösungen sind in Wirklichkeit Scheinlösungen oder beruhen auf Unkenntnis:

- Der sogenannte „modal split“ (also die Kennzahl über die relativen Anteile der Verkehrsträger) sagt nichts über die realen ökologischen und sozialen Belastungen aus.

- Die Fixierung der Betrachtung auf die Luftschadstoffemissionen heißt andere Wirkungen, wie Lärm, Flächenverbrauch, Verkehrssicherheit etc. zu unrecht zu vernachlässigen.

- Die Konzentration auf den bedingungslosen Ausbau des öffentlichen Verkehrs ist nicht per sé positiv und schon gar nicht zwangsläufig ökologisch.

- Häufig werden Maßnahmen als Innovationen verkauft, die originäre Aufgaben der verkehrlichen Politikstrategien darstellen.
- Bisher fehlt es in den Politikstrategien an der konsequenten Entwicklung von Verkehrsvermeidungsansätzen, die vor allem auch Siedlungsund Wirtschaftsstrukturen berücksichtigen.

Das zugrundeliegende Problem ist aber nicht nur ein Umsetzungsproblem in der Verkehrsplanung, sondern hat scheinbar mit dem spezifischen Charakter von Mobilität und Verkehr zu tun: Es fehlt das Politikmodell, das den vielschichtigen Verästelungen zwischen dem Verkehr und den mobilitätsbestimmenden Randbedingungen gerecht werden kann.

\section{- Konsequenzen}

Wie kann die kommunale Verkehrsplanung angemessen darauf reagieren? Zunächst sollte geklärt werden, wie nachhaltiger Verkehr auf der Strategieebene erreicht werden könnte. Wichtig erscheint vor allem eine Versachlichung (Gegenbeispiel: die fünf-Mark-Kampagne) in der Auseinandersetzung mit dem Handlungsfeld.

\section{- Mobilität als Verteilungskonflikt}

Offensichtlich ist der Verkehr ein tragendes Element von Wettbewerb - Verkehr bestimmt die Konkurrenz der privaten Lebensentwürfe, er beeinflußt die Marktmacht der Unternehmen und den (Standort-) Wettbewerb der Städte und Regionen um die Gunst des Kapitals. Jeder Versuch, auf die eigendynamische Verkehrsentwicklung mit Planung, Gestaltung und Restriktion zu reagieren, muß solange scheitern, wie er auf die Verteilung dieser Chancen einseitig Einfluß nimmt und die faire Ausgestaltung des Rahmens nicht erfolgt.

Außerdem wird die Einsicht in die Tatsache notwendig sein, daß nicht beides möglich ist: sowohl ,immer schneller und besser zu leben“ als auch nicht negativ durch die Folgen des KfzVerkehrs belastet zu werden.

\section{- Mobilität als Medium}

Es bedarf der richtigen Strukturen (Raum, Zeit, Bauten), die eine individuelle Anpassung ermöglichen und mit einem geringeren (Gesamt-)Verkehrsaufwand einhergehen. Schließlich ist Mobilität in den wenigsten Fällen Selbstzweck.

\section{- Mobilität als Innovationsfeld}

Es haben sich zwar, wie beschrieben, die Ziele der Verkehrsplanung in den vorangegangenen Jahren verändert, nicht aber ihre Methoden. Ein Methodenwandel als dritte Plangeneration hätte umfangreiche Konsequenzen für Planorganisation, Personalstrukturen und Berufsbilder (4). Ein neues Verständnis von Mobilität und Verkehr schafft die Grundlage für die notwendige Entwicklung neuer Angebotsformen (Mobilitätsdienstleistungen) als Alternative zum motorisierten Individualverkehr.

- Flankierung verkehrlicher Politikstrategien Kommunale Politikstrategien im Bereich Mobilität/ Verkehr müssen auf Landes- und Bundesebene flankiert werden. Neben indirekten Einflußfaktoren (Steuern, finanzielle Förderung) wirken verschiedene planerische und gesetzliche Instrumente (Bundesverkehrswegeplanung, Landesentwicklungsprogramm) direkt auf die Verkehrsentwicklung.

- Mobilität als Gegenstand politischer Regulierung

Auf eine politische Regulierung, beispielsweise in Form von Orten und Verfahren zur gemeinsamen Gestaltung und Austragung unterschiedlicher Auffassungen und immanenter Interessenkonflikte, wird nicht verzichtet werden können. Schließlich sind die unterschiedlichen Anforderungen der verschiedenen Anspruchsgruppen in wenigen Bereichen so ausdifferenziert wie im Bereich Mobilität. Hierbei spielt die Klärung von Verfahrensfragen eine mindestens ebenso wichtige Rolle wie die Konzepte und Maßnahmen selbst.

\section{Anmerkungen}

(1) Der Beitrag beruht auf ersten Zwischenergebnissen einer Studie im Auftrag der Hans-Böckler-Stiftung, und gemeinsamen Überlegungen mit Markus Hesse. Ziel der Studie ist es, Grundlagen eines Leiffadens für lokale und regionale Politikstrategien im Bereich "Mobilitöt" zu entwickeln.

(2) Appel, Heinz Peter, Reinhold Baier: Methoden und Ablauf der kommunalen Verkehrsplanung. In: Apel, Dieter u.a. (Hrsg.): Handbuch der kommunalen Verkehrsplanung. Economica Verlag, Bonn, Grundwerk 1992. Kapitel 3.2.10.1., S.10-48.

(3) Würdemann, Gerd: Einführung. Suche nach einem umsetzbaren Konzept. In: Bundesforschungsanstalt für Landeskunde und Raumordnung (Hrsg.): Ballungsraumverkehr. Integrierte Verkehrsplanung und Verkehrsvermeidung. Informationen zur Raumentwicklung Heft 7/8.1996. S.VII. (4) Vgl. Petz, Peter: Zufußgehen und Rodfahren. Auf dem Weg zu einer "Dritten Verkehrsplangeneration“. RaumPlanung 79,1997, S.258-266.

Die Autorin
Heike Flämig betreut das Forsthungsfeld Stadtent-
wicklung, Planung, Verkehr in I0̈W Berlin.
Kontakt: 10 W, Giesebrechistr. 13,10629 Berlin.
Tel. $030 / 884594-0$, Fax $030 / 8825439$,
E-mail: Heike.Flaemig@ioew.b.eunet.de


(c) 20I0 Authors; licensee IÖW and oekom verlag. This is an article distributed under the terms of the Creative Commons Attribution Non-Commercial No Derivates License (http://creativecommons.org/licenses/by-nc-nd/3.o/), which permits unrestricted use, distribution, and reproduction in any medium, provided the original work is properly cited. 